\title{
Differential matrix metalloproteinase levels in adenocarcinoma and squamous cell carcinoma of the lung
}

\author{
Sonam A. Shah, BS, Francis G. Spinale, MD, PhD, John S. Ikonomidis, MD, PhD, Robert E. Stroud, MS, \\ Eileen I. Chang, PhD, and Carolyn E. Reed, MD
}

Objective: The matrix metalloproteinases (MMPs) have been implicated in the aggressive course of non-small cell lung cancer (NSCLC). However, there are a large number of MMP subtypes with diverse proteolytic substrates and different induction pathways. This study tested the hypothesis that a differential MMP profile would exist between NSCLC and normal lung and that MMP patterns would differ between NSCLC histologic types.

\begin{abstract}
Methods: NSCLC samples and remote normal samples were obtained from patients with stage I or II NSCLC with either squamous cell $(n=22)$ or adenocarcinoma $(n=19)$ histologic characteristics. Absolute concentrations for each of the MMP subclasses were determined by a calibrated and validated multiplex suspension array: collagenases (MMP-1, -8, and -13), gelatinases (MMP-2 and -9), lysins (MMP-2 and -7), and elastase (MMP-12).
\end{abstract}

Results: Overall, MMP levels were significantly increased in NSCLC compared with normal. For example, MMP-1 and MMP-7 increased by approximately 10 -fold in NSCLC $(P<.05)$. Moreover, a different MMP portfolio was observed between NSCLC histologic types. For example MMP-1, $-8,-9$, and -12 increased by more than 4 -fold in squamous cell versus adenocarcinoma $(P<.05)$. In those patients who had recurrence within 3 years of resection, 3 -fold higher levels of MMP-8 and -9 were observed $(P<.05)$.

Conclusion: Increased levels of a number of MMP types occur with NSCLC, but the MMP profile was distinctly different between histologic types and in those patients with recurrence. These different MMP profiles may be important in the mechanistic basis for the natural history of different NSCLC types, as well as identifying potential prognostic and therapeutic targets. (J Thorac Cardiovasc Surg 2010;139:984-90)

Matrix metalloproteinases (MMPs) are a large family of structurally and functionally related zinc endopeptidases that collectively can degrade virtually all extracellular matrix components and can regulate the tumor microenvironment through processing substrates including growth factors and their receptors, cell adhesion molecules, cytokines, chemokines, apoptotic ligands, and angiogenic factors. $^{1,2}$ The potential role and regulation of MMPs have been the subject of a number of past studies in lung cancer. ${ }^{1-12}$ However, these past studies usually focused on a single MMP type or class, and therefore the pattern of MMP expression in lung cancer remained poorly understood. Moreover, although the diversity and complexity of the MMP family are becoming widely recognized, the initial

\footnotetext{
From the Division of Cardiothoracic Surgery, Medical University of South Carolina and the Ralph H. Johnson VA Medical Center, Charleston, SC.

This study was supported by National Institutes of Health grants HL59165 and HL81691 and by a Merit Award from the Veterans' Affairs Health Administration. S.A.S. participated in this project as a recipient of a medical student fellowship award from The American Association of Thoracic Surgery.

Disclosures: None.

Read at the Eighty-ninth Annual Meeting of The American Association for Thoracic Surgery, Boston, Massachusetts, May 9-13, 2009.

Received for publication May 4, 2009; revisions received Nov 5, 2009; accepted for publication Dec 12, 2009.

Address for reprints: Carolyn E. Reed, MD, Department of Surgery, MUSC, 25 Courtenay Dr, Charleston, SC 29425 (E-mail: reedce@musc.edu).

0022-5223/\$36.00

Copyright (c) 2010 by The American Association for Thoracic Surgery doi: $10.1016 /$ j.jtcvs.2009.12.016
}

strategy for MMPs in the context of cancer therapeutics was that of broad-spectrum, global MMP inhibitors. ${ }^{13-16}$ These initial broad-spectrum MMP inhibitors were not successful, and the underlying reasons for this were multifactorial and likely included poor specificity and adverse side effects. One outcome from these past MMP pharmacologic studies was the recognition that more targeted therapeutic approaches would be required. Thus, studies that more carefully quantify a more diverse number of MMPs in the context of cancer, such as non-small cell lung cancer (NSCLC), are warranted. Accordingly, the overall goal of the present study was to quantify a large number of MMP types, from different MMP classes, in samples taken from NSCLC and compare these with normal regions from the same patient. Moreover, this study examined whether and to what degree different MMP portfolios would emerge in NSCLC of different histologic types and in patients with early recurrence.

\section{MATERIALS AND METHODS Patients}

Snap-frozen lung specimens were taken from the targeted resection region from patients with either stage I or II NSCLC (22 squamous cell; 19 adenocarcinoma) or from the remote, normal region. These samples were retained under cryogenic conditions in the Hollings Cancer Center, Medical University of South Carolina tissue repository. The adenocarcinoma cohort consisted of 16 patients with stage I and 3 patients with stage II (4 men; 15 women). The squamous cell cohort included 16 patients with stage I and 6 patients with stage II (18 men, 4 women). Before tissue sampling at the time 


\section{Abbreviations and Acronyms \\ MMP = matrix metalloproteinase \\ NSCLC $=$ non-small cell lung cancer \\ TIMP $=$ tissue inhibitors of the matrix metalloproteinase}

of tumor resection, all patients had signed an informed consent form for tissue banking purposes, and this specific study was approved by the Institutional Review Board of the Medical University of South Carolina, Charleston, South Carolina (HR\# 18229). The data obtained from these banked samples included the histologic type (squamous cell carcinoma, adenocarcinoma) as well as whether recurrence had occurred within 3 years of the initial resection. Recurrence was defined as locoregional (recurrence of tumor within the lung or mediastinal lymph nodes ipsilateral to the side of surgery) or distant.

\section{Sample Preparation}

The lung samples were processed in a simultaneous fashion using a homogenization and extraction protocol that has been demonstrated previously to successfully remove both high and low molecular weight proteins, such as MMPs, from solid tissue samples. ${ }^{17-19}$ This method uses a homogenization process, an extraction buffer, and a centrifugation method by which a complete uniform yield of intracellular and extracellular protein is obtained. In brief, a uniform weight $(50 \mathrm{mg})$ of the frozen samples was placed in ice-cold extraction/homogenization buffer (buffer volume used was $1: 6 \mathrm{w} / \mathrm{v}$, containing $10 \mathrm{mmol} / \mathrm{L}$ cacodylic acid $\mathrm{pH} 5.0,0.15 \mathrm{~mol} / \mathrm{L}$ $\mathrm{NaCl}, 20 \mathrm{mmol} / \mathrm{L} \mathrm{ZnCl}, 1.5 \mathrm{mmol} / \mathrm{L} \mathrm{NaN}_{3}$, and $0.01 \%$ Triton X-100 [v/ v]) and allowed to thaw on ice. $\mathrm{We}^{18,20}$ have demonstrated previously that this approach stabilizes proteins and proteases during the extraction process. Stainless steel beads $(5 \mathrm{~mm})$ were placed in the samples, and the beads were oscillated at a high frequency $(30 \mathrm{~Hz} ; 2$ cycles, 5 minutes each), resulting in full tissue disruption and uniform homogenization (TissueLyserII, catalog No. 85300; Qiagen NV, Venlo, The Netherlands). This homogenization approach uses a full cell disruption technique to fully extract intracellular and extracellular proteins from a solid tissue sample. The homogenate was then centrifuged $\left(800 \mathrm{~g}, 10\right.$ minutes, $4^{\circ} \mathrm{C}$ ) (model 5810 or $5417 \mathrm{c}$; Eppendorf AG, Westbury, NY) and the supernatant removed to a fresh tube and stored on ice. Protein concentrations were determined in duplicate from the final extracts by the Bradford method (Bio-Rad Protein Assay; Bio-Rad Laboratories, Hercules, Calif) and then titrated to a uniform protein concentration $(100 \mu \mathrm{g})$, aliquoted, and stored at $-80^{\circ} \mathrm{C}$ until subjected to multiplex suspension array analysis.

\section{Analytical Measurements}

The protein titrated samples were thawed on ice and subjected to multiplex suspension array (Human Fluorokine MAP MMP Kit, LMP000; R\&D Systems, Inc, Minneapolis, Minn), in which all samples could be measured in simultaneous fashion, thereby minimizing interassay coefficient of variation. The titrated samples were subjected to this assay in triplicate, and these triplicate values were then examined with respect to intra-assay coefficient of variation. For repeated samples that were outside a $15 \%$ confidence interval ( 3 occurrences in the present study), the entire homogenization and assay were repeated. The multiplex array was previously validated and calibrated using internal controls for each MMP type. $^{21}$ The matrix was assembled to measure representative subtypes from each class of MMPs that included the collagenases (MMP-1, MMP8, and MMP-13), the gelatinases (MMP-2 and MMP-9), the stromelysins/ matrilysins (MMP-3/MMP-7), and the elastases (MMP-12). The relative fluorescence obtained for each distinct MMP (Bio-Plex 200; Bio-Rad Laboratories) was converted to an absolute concentration using standards that
TABLE 1. Multiplex matrix metalloproteinase (MMP) antisera and sensitivity

\begin{tabular}{llll}
\hline Analyte & Manufacturer & Catalog no. & Sensitivity \\
\hline MMP-1 & R\&D Systems & LMP901 & $4.44 \mathrm{pg} / \mathrm{mL}$ \\
MMP-2 & R\&D Systems & LMP902 & $25.4 \mathrm{pg} / \mathrm{mL}$ \\
MMP-3 & R\&D Systems & LMP513 & $1.3 \mathrm{pg} / \mathrm{mL}$ \\
MMP-7 & R\&D Systems & LMP907 & $16.9 \mathrm{pg} / \mathrm{mL}$ \\
MMP-8 & R\&D Systems & LMP908 & $8.9 \mathrm{pg} / \mathrm{mL}$ \\
MMP-9 & R\&D Systems & LMP911 & $7.4 \mathrm{pg} / \mathrm{mL}$ \\
MMP-12 & R\&D Systems & LMP919 & $1.2 \mathrm{pg} / \mathrm{mL}$ \\
MMP-13 & R\&D Systems & LMP511 & $15.9 \mathrm{pg} / \mathrm{mL}$ \\
\hline
\end{tabular}

were included in each assay, and the specifications for each reagent and sensitivity are shown in Table 1 . The coefficient of variation for these assays was $15 \%$ or less. For all of the MMP multiplex assays, the sample readings fell within the targeted dynamic range for the analyte of interest. A representative standard curve and the sample results for MMP-2 are shown in Figure 1. All MMP assays were blinded to region, histology, and status of recurrence.

\section{Data Analysis}

The fluorescence emissions for each set of MMP standards were first fit to a 5-parameter logistic equation (Figure 1), following a conventional format for nonlinear fluorescence profiles. ${ }^{22}$ Using this 5-parameter logistic curve fit, the fluorescence emissions for the samples were converted to actual MMP values and then finally to MMP content per milligram of wet sample weight. The comparisons of MMP profiles by region and by histologic type, pair-wise comparisons, were performed with a $t$ statistic. For the purposes of comparisons of MMP profiles by both region and histologic type analysis of variance was performed in which region and histologic type were

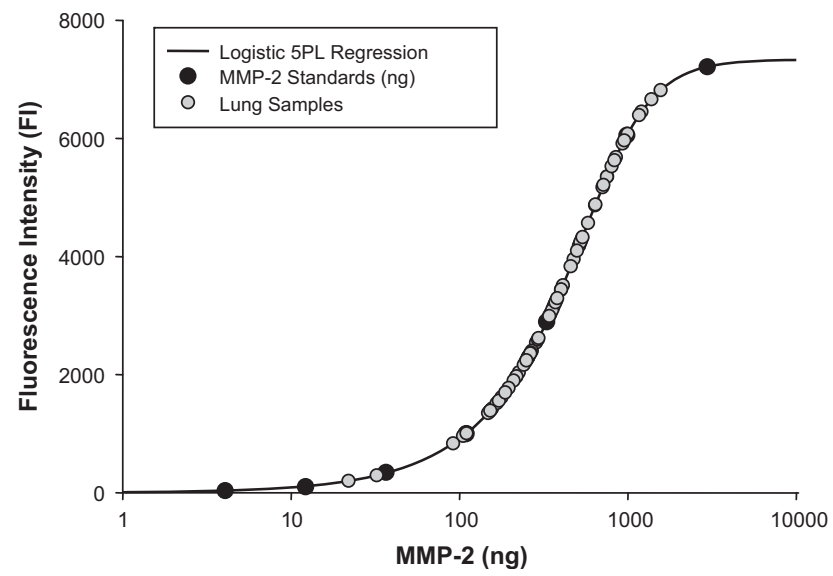

FIGURE 1. A representative 5-parameter logistic (5-PL) curve developed for MMP-2 using a wide range of MMP-2 recombinant control standards (black circles). The 5-PL uses the equation where $\mathrm{y}=\mathrm{d}+(\mathrm{a}-\mathrm{d}) /(1+(\mathrm{x} /$ c) $\left.)^{\wedge}\right)^{\wedge} \mathrm{g}$ ), in which $x=$ concentration of standard, $y$ is the response variable, $a$ is the estimated response at 0 concentration, $b$ is the slope of the tangent midpoint, $c$ is the midrange of concentration or midpoint, $d$ is the estimated response at infinite concentration, and $g$ is an asymmetry factor. This nonlinear regression fitting is optimal for response variables such as fluorescence emission. $^{22}$ The lung samples that were loaded using identical protein concentrations (gray circles) all fell within the dynamic range of the standards and the 5-PL equation. 
TABLE 2. MMP content in normal and NSCLC specimens

\begin{tabular}{|c|c|c|c|c|c|c|}
\hline \multirow[b]{2}{*}{ MMP type } & \multicolumn{2}{|c|}{ Region } & \multirow[b]{2}{*}{$P$ value } & \multicolumn{2}{|c|}{ Histologic type } & \multirow[b]{2}{*}{$P$ value } \\
\hline & Normal & NSCLC & & AdenoCa & Sq Cell Ca & \\
\hline \multicolumn{7}{|l|}{ Collagenases } \\
\hline MMP-1 & $1.3 \pm 0.9$ & $33.7 \pm 10.9$ & .0025 & $8.3 \pm 2.4$ & $55.7 \pm 19.2$ & .0115 \\
\hline MMP-13 & $0.1 \pm 0.1$ & $7.9 \pm 5.3$ & .0731 & $1.8 \pm 0.6$ & $13.1 \pm 9.8$ & .1347 \\
\hline MMP-8 & $85.2 \pm 10.8$ & $296.4 \pm 84.0$ & .0084 & $53.3 \pm 22.6$ & $506.4 \pm 141.9$ & .0023 \\
\hline \multicolumn{7}{|l|}{ Gelatinases } \\
\hline MMP-2 & $57.3 \pm 7.5$ & $161.0 \pm 58.8$ & .0437 & $79.7 \pm 20.3$ & $231.3 \pm 107.0$ & .0888 \\
\hline MMP-9 & $216.3 \pm 24.3$ & $185.5 \pm 37.8$ & .2479 & $89.5 \pm 28.6$ & $268.4 \pm 61.4$ & .0065 \\
\hline \multicolumn{7}{|l|}{ Lysins } \\
\hline MMP-3 & $0.7 \pm 0.1$ & $38.6 \pm 30.8$ & .1130 & $1.3 \pm 0.4$ & $70.8 \pm 57.1$ & .1183 \\
\hline MMP-7 & $7.9 \pm 1.5$ & $75.1 \pm 15.8$ & .0001 & $87.4 \pm 23.0$ & $63.5 \pm 21.9$ & .4559 \\
\hline \multicolumn{7}{|l|}{ Elastases } \\
\hline MMP-12 & $1.4 \pm 1.2$ & $24.4 \pm 6.5$ & .0006 & $7.8 \pm 2.9$ & $38.7 \pm 11.0$ & .0062 \\
\hline Sample size (n) & 41 & 41 & & 19 & 22 & \\
\hline
\end{tabular}

$M M P$, Matrix metalloproteinase; $N S C L C$, non-small cell lung cancer; AdenoCa, adenocarcinoma; $S q C e l l C a$, squamous cell carcinoma. MMP types are presented by MMP class.

*Values reported in picograms per milligram wet sample weight.

considered main treatment effects. For the purposes of examining the relative MMP profiles to recurrence rates, a categorical analysis was performed. First, the samples were stratified to recurrence or no recurrence and the relative MMP values compared between these stratifications. Inasmuch as these data were not normally distributed, a Wilcoxon test was performed. All statistical procedures were performed with STATA statistical software (STATA Intercooled version 8.0; Stata, Inc, College Station, Tex). Results are presented as mean \pm standard error of the mean.

\section{RESULTS}

The summary of MMP levels, by region (normal, NSCLC) and by histologic type, is presented in Table 2 . The MMP types examined were categorized by subgroups as shown. For the MMP collagenase subgroup, MMP-1 and MMP-8 increased by more than 3-fold. Relative levels for MMP-13 appeared increased in the NSCLC region, but owing to a high degree of variation between samples, this did not reach statistical significance. For the MMP

TABLE 3. Analysis of variance results for main effects (region and histology) and interactions

\begin{tabular}{|c|c|c|c|c|c|c|}
\hline \multirow[b]{2}{*}{ MMP type } & \multicolumn{2}{|c|}{ Region } & \multicolumn{2}{|c|}{ Histology } & \multicolumn{2}{|c|}{ Interaction } \\
\hline & F-statistic & $P$ value & F-statistic & $P$ value & F-statistic & $P$ value \\
\hline \multicolumn{7}{|l|}{ Collagenases } \\
\hline MMP-1 & 8.67 & .0043 & 5.58 & .0206 & 4.71 & .0330 \\
\hline MMP-13 & 1.97 & .1644 & 1.11 & .2955 & 1.11 & .2961 \\
\hline MMP-8 & 6.34 & .0138 & 10.16 & .0021 & 6.91 & .0103 \\
\hline \multicolumn{7}{|l|}{ Gelatinases } \\
\hline MMP-2 & 2.83 & .0966 & 2.21 & .1413 & 1.18 & .2801 \\
\hline MMP-9 & 0.71 & .4037 & 5.67 & .0197 & 3.07 & .0837 \\
\hline \multicolumn{7}{|l|}{ Lysins } \\
\hline MMP-3 & 1.32 & .2541 & 1.30 & .2582 & 1.26 & .2653 \\
\hline MMP-7 & 19.04 & .0000 & 0.34 & .5593 & 0.91 & .3444 \\
\hline \multicolumn{7}{|l|}{ Elastases } \\
\hline MMP-12 & 12.45 & .0007 & 7.07 & .0095 & 5.34 & .0235 \\
\hline
\end{tabular}

$M M P$, Matrix metalloproteinase. gelatinase subgroup, MMP-2 levels were increased by approximately 3-fold in the NSCLC region. In the lysin and the elastase subgroups, MMP-7 and MMP-12, respectively, were increased by more than 10-fold in the NSCLC region. Moreover, a different MMP portfolio was observed between NSCLC histologic types. For example, MMP-1, -8, -9, and -12 increased by more than 4 -fold in squamous cell versus adenocarcinoma. In light of the fact that MMP levels changed as a function of region (normal, NSCLC) and histologic type, analysis of variance was performed to identify main effects and potential interactions (Table 3). From this analysis, it was demonstrated that a significant region and histologic type interaction occurred for MMP-1, -8, and -12 , with MMP-9 approaching statistical significance. This analysis further confirmed the univariate analysis in that changes in the levels of specific MMP types were affected by NSCLC as well as by histologic type. Finally, MMP levels in patients whose tumor did not recur were compared with MMP concentrations in patients whose tumor did recur within 3 years (Table 4). Recurrence was evenly divided between distant and locoregional. From this approach, increased levels for MMP-8 and MMP-9 were observed within the NSCLC region in those patients with recurrence.

\section{DISCUSSION}

The MMPs constitute a large family of proteolytic enzymes, which were historically considered to have similar proteolytic portfolios and induction pathways. However, it is now recognized that specific MMP types can be induced by an array of biological signaling pathways and biophysical events and that this induction is not a uniform process. Moreover, the profile for MMPs, in general, is highly diverse, and specific MMPs can exhibit unique biological effects on extracellular matrix proteins, cell adhesion, cytokine processing, and cell growth characteristics. Much 
TABLE 4. Recurrence and tumor MMP content*

\begin{tabular}{lccc}
\hline \multicolumn{1}{c}{ MMP type } & No recurrence & Recurrence & $\boldsymbol{P}$ value \\
\hline Collagenases & & & \\
$\quad$ MMP-1 & $30.3 \pm 12.2$ & $43.2 \pm 24.4$ & .5963 \\
MMP-13 & $1.4 \pm 0.4$ & $25.5 \pm 19.5$ & .3977 \\
$\quad$ MMP-8 & $148.2 \pm 37.4$ & $700.4 \pm 268.0$ & .0057 \\
Gelatinases & & & \\
$\quad$ MMP-2 & $97.6 \pm 18.5$ & $334.1 \pm 211.3$ & .3315 \\
MMP-9 & $132.0 \pm 22.9$ & $331.4 \pm 119.4$ & .0158 \\
Lysins & & & \\
MMP-3 & $6.2 \pm 2.3$ & $126.7 \pm 114.1$ & .2392 \\
$\quad$ MMP-7 & $83.7 \pm 20.2$ & $50.3 \pm 18.0$ & .6995 \\
Elastases & & & \\
$\quad$ MMP-12 & $25.6 \pm 8.5$ & $21.0 \pm 7.4$ & .5562 \\
Sample size (n) & 30 & 11 & \\
\hline
\end{tabular}

$M M P$, Matrix metalloproteinase. *Values reported in picograms per milligram wet sample weight.

of this new insight into MMP biology has been brought about by past studies that have examined MMP regulation and expression patterns in cancer, and more specifically lung carcinoma. ${ }^{1,2,13}$ However, past clinical studies that have examined relative MMP expression and/or levels in lung cancer specimens have focused on one MMP type or a single class of MMPs. ${ }^{3-12}$ In addition, past studies have used semiquantitative approaches or examined MMP levels in relative terms, rather than in absolute levels. As a consequence, the absolute changes in MMP levels across a wide spectrum of MMP types and classes in a focused set of lung cancer specimens, such as NSCLC, remained unknown. Accordingly, the present study used a quantitative approach to measure absolute MMP levels from all of the classes of soluble MMP types in NSCLC and normal lung samples. The unique findings from this study were 2-fold. First, a diverse number of MMP types are increased in absolute amounts in NSCLC, and these levels change as a function of histologic type. Second, the absolute levels for certain MMP types were increased in samples taken from patients with tumor recurrence. Taken together, these findings demonstrate that a full portfolio of MMP types can be consistently measured in NSCLC samples, which may hold both prognostic and therapeutic relevance.

A number of past studies have successfully isolated RNA and protein from tumor samples and profiled a limited number of MMPs. ${ }^{23-26}$ For example, protein was successfully extracted from thyroid tumors, and relative MMP-1 and MMP9 measurements were performed using a targeted enzymelinked immunoassay. ${ }^{27}$ This past study demonstrated an association between higher levels of MMP-1 and MMP-9 to thyroid histologic type. The present study used a reproducible protein extraction method and a unique MMP multiplex approach that allowed for quantification of 8 individual MMP protein levels simultaneously. Through this quantitative approach, the results demonstrated important differences in absolute MMP abundance profiles between
NSCLC and normal lung samples, as well as between distinct NSCLC histologic profiles. For example, absolute levels of MMPs within the collagenase class (MMP-1, -8 , and -13) increased significantly in NSCLC, and increased levels of MMP-1 and MMP-8 were particularly pronounced in squamous cell carcinoma samples. Past studies have identified that this class of MMPs can play a role in a number of biological processes relevant to cancer progression, which includes growth, migration, invasion, apoptosis, and angiogenesis. ${ }^{1-5,25,26}$ However, a number of considerations must be taken into account when evaluating absolute MMP levels and to what extent these levels may be reflective of changes in overall proteolytic potential and thereby influencing relevant biological events. In general terms, net MMP proteolytic activity is dependent on transcriptional and posttranslational events, which include MMP synthesis, secretion, and activation. ${ }^{1,2}$ The present study demonstrated that absolute levels of specific MMP types changed in NSCLC, but it could not differentiate between the proform and active form of these MMPs. In past immunohistochemical studies, a similar limitation existed whereby changes in the relative abundance of certain MMP types could be identified in a tumor specimen, but the activational state of the MMP could not be discerned..$^{-7,10,11}$ Although the net changes in proteolytic activity could not be determined in the present study, the absolute changes in MMP levels that were observed in the NSCLC samples are likely reflective of changes in upstream induction pathways. Specifically, MMPs are tightly regulated at the transcriptional level, ${ }^{28,29}$ in which both ligand and adhesion mediated receptor interactions alter intracellular formation of transcription factors that specifically bind to regulatory elements within the MMP promoter region. Thus, the absolute changes in MMP profiles with NSCLC that were observed in the present study are likely the result of altered transcription pathways that are reflective of the underlying cancer process. Indeed, MMP expression profiles have been demonstrated to be directly associated with the relevant process of tumor biology, which includes local growth and prognosis. ${ }^{1,5,7,11,23,25,26}$ The present study provides for a proof of concept that profiling of a large array of MMPs in NSCLC is possible and could be expanded to reveal specific subsets of MMPs that hold both diagnostic and therapeutic relevance.

Whereas past studies have examined one or several MMP types in NSCLC specimens, ${ }^{3-12}$ these past investigations have used semiquantitative approaches; therefore, direct comparisons to the present study are difficult, where absolute protein levels of a full spectrum of MMP types were quantified in normal and NSCLC regions. However, in general, past studies have identified that a relative increase in MMP-1, -2, -7, and -12 has occurred in NSCLC, consistent with the current study. ${ }^{3-12}$ Moreover, in the present study, a differential MMP profile could be demonstrated between histologic types of NSCLC, which can be difficult when 
using immunohistologic and morphometric approaches. In the present study, absolute levels of MMP-9 were increased in squamous cell carcinoma but not in adenocarcinoma. A past study by Pinto and associates ${ }^{10}$ suggests that the increased levels of MMP-9 in this histologic type of NSCLC can be associated with a poor prognosis. Taken together, these results suggest that simultaneous quantitative measurements of a full portfolio of MMPs in NSCLC specimens may provide for distinct tumor profiling and thereby develop specific thresholds for MMP protein levels for prognostic use. However, further examination of how MMP profiles differ between histologic types of NSCLC and are related to prognosis will be required. Furthermore, the results from the present study using MMP protein measurements with a high sensitivity multiplex array will need to be performed in parallel with histologic studies to provide cell and spatial information. For example, Thomas and associates ${ }^{11}$ demonstrated a higher expression of certain MMP types in adenocarcinoma using morphometric scoring of immunohistochemical stained specimens. Furthermore, inasmuch as the multiplex array provides for a high sensitivity approach for measuring MMPs in small sample volumes, then future studies that examine the relationship between MMP levels in NSCLC samples to that of a simultaneously obtained plasma sample would be of prognostic relevance. Indeed, initial studies of profiling of MMPs in a collected blood sample have shown prognostic potential in several different cancers. ${ }^{30-35}$ For example, plasma MMP levels (particularly MMP-2, -7, and -9) have been studied in a variety of cancers, including colon cancer, ${ }^{30,31}$ breast cancer, ${ }^{32}$ gastric cancer, ${ }^{33}$ and renal cell carcinoma. ${ }^{34}$ Moreover, increased circulating MMP-9 levels have been identified in patients with NSCLC. ${ }^{35}$ Indeed, the relative increase in plasma MMP-9 levels reported in this past study parallel the magnitude of absolute changes in MMP-9 levels that were observed in the present study with respect to the squamous cell NSCLC samples. However, these past studies and the present study only provide associative results, and a prospective study that simultaneously measures the relationship between circulating and tumor levels of MMPs in patients with NSCLC to that of clinical outcomes would be warranted.

The present study measured absolute levels of MMPs in NSCLC samples and identified that certain MMPs changed dramatically whereas other MMP types remained unaltered when compared with normal samples. In addition, the levels of certain MMP types appeared to be selectively increased in certain histologic types. Through this type of broad MMP profiling, it may be possible to identify which MMPs may be specifically expressed in NSCLC, as well as in a specific histologic type, and thereby allow for the formation of hypothesis-based, directed pharmacologic studies. Although MMPs in general have been identified as potential targets for anticancer pharmacology, the use of broad-spectrum MMP inhibitors has not met with success. ${ }^{13-16}$ Postulated reasons for the failure of broad-spectrum MMP inhibition in the context of NSCLC are that inhibition of certain MMP types that are not contributory to cancer progression will result in toxic side effects, initiation or progression of disease, and poor patient outcome. In the present study, absolute levels of MMP-7 were increased by 10-fold in NSCLC, and this MMP type has been identified for more specific pharmacologic inhibition in the context of cancer progression. ${ }^{13,27,36}$ MMP-7 is one of only a few MMPs actually secreted by tumor cells. It regulates cell proliferation and apoptosis by cleaving the ectodomain of heparin binding-epidermal growth factor precursor. ${ }^{1}$ MMP-7 cleaves E-cadherin, a cell-adhesion molecule, and deregulation of E-cadherin is associated with cancer progression. ${ }^{2}$ Cleavage also triggers the epithelial-to-mesenchymal transition. However, the relationship between the biological function of certain MMP types and the quantitative measurements performed in the present study remain associative at best. Nevertheless, owing to the unique functionality of certain MMP types and the marked overexpression pattern observed in the present set of NSCLC specimens, a postulate for further study is that pharmacologic regulation of certain MMP types is required in certain forms of NSCLC and that MMP profiling would provide a means to tailor pharmacotherapy. The differential profile observed in the present study and the diversity of biological functions of different MMP types underscores a likely reason for disappointing results when broad-spectrum MMP inhibitors were evaluated. ${ }^{14-16,27,36}$

MMP-2, ${ }^{4,9}$ MMP-7,,12 MMP-9,,,${ }^{4,610}$ and MMP-12, $2^{7,8}$ overexpression have been related to poor outcome in patients with NSCLC. However, different analysis techniques, semiquantitative analyses, and differing stages and histologic types make comparisons difficult. We found increased levels of MMP-8 and MMP-9 in patients who had recurrence within 3 years of surgery compared with those who did not (Table 4). Overexpression of several MMPs tested (MMP-2, MMP-3, MMP-9, and MMP-13) have been associated with epithelial-to-mesenchymal transition, a fundamental biological process in which epithelial cells lose their polarity, cell-cell adhesion, and adopt a mesenchymal morphology appropriate for migration. ${ }^{1}$ Tumor staining for MMP-9 in resected adenocarcinoma of the lung has been shown to be strongly related to survival. ${ }^{10}$ In another study, detection of homogeneous MMP-9 in NSCLC cells was an independent prognostic factor for shortened cancer-related survival. ${ }^{6}$ More patients with recurrence must be studied to ascertain whether an MMP expression profile can be constructed that will predict early recurrence of resected adenocarcinoma and squamous cell carcinoma of the lung.

\section{LIMITATIONS}

Several limitations of the present study must be recognized. First, this study quantified MMP types in a small number of samples from squamous and adenocarcinoma 
NSCLC. Thus, the study was underpowered to perform a number of relationships between tumor biology and outcomes. Nevertheless, our preliminary results suggest that a relationship between recurrence and certain MMP levels is likely to exist in the context of NSCLC. Second, more than 26 MMP types have been described, and the present study therefore only examined a limited number. However, this study constructed the multiplex array to encompass representative MMPs from the major classes of MMP types and those that have been reported in past NSCLC histologic studies. Future studies that expand the sample size and MMP portfolio would be appropriate. Third, a critical control point of MMP activity is the tissue inhibitors of the MMPs (TIMPs) and can regulate MMP activity by binding MMPs in a 1:1 stoichiometry. The biological functions of the TIMPs are also complex. ${ }^{37}$ For example, elevated levels of TIMP-1 have been associated with a poor prognosis in NSCLC. ${ }^{38,39}$ Thus, a future study that builds on the MMP multiplex assay through quantification of the 4 known TIMPs would be an important future direction. Fourth, this study measured MMP profiles from whole lung samples, and therefore the specific cell types and relative MMP distribution throughout the tumor could not be determined. Nevertheless, the present study demonstrated quantitative differences in MMP levels of early-stage adenocarcinoma and squamous cell carcinoma, as well as tumors that have recurred within 3 years of resection. These new results underscore the diversity of MMPs in NSCLC and suggest that profiling this proteolytic system would hold clinical significance.

\section{References}

1. Noël A, Jost M, Maquoi E. Matrix metalloproteinases at cancer tumor-host interface. Sem Cell Dev Biol. 2008;19:52-60.

2. Egebad M, Werb Z. New functions for the matrix metalloproteinases in cancer progression. Nat Rev Cancer. 2002;2:161-74.

3. Pritchard SC, Nicolson MC, Lloret C, McKay JA, Ross VG, Kerr KM, et al. Expression of matrix metalloproteinases 1, 2, 9 and their tissue inhibitors in stage II non-small cell lung cancer: implications for MMP inhibition therapy. Oncol Rep. 2001;8:421-4.

4. Kodate M, Kasai T, Hashimoto H, Yasumoto K, Iwata Y, Manabe H. Expression of matrix metalloproteinase (gelatinase) in T1 adenocarcinoma of the lung. Pathol Int. 1997;47:461-9.

5. Leinonen T, Pirinen R, Böhm J, Johansson R, Ropponen K, Kosma V-M. Expression of matrix metalloproteinases 7 and 9 in non-small cell lung cancer. Relation to clinicopathological factors, $\beta$-catenin and prognosis. Lung Cancer. 2006;51: 313-21.

6. Sienel W, Hellers J, Morresi-Hauf A, Lichtinghagen R, Mutschler W, Jochum M, et al. Prognostic impact of matrix metalloproteinase-9 in operable non-small cell lung cancer. Int J Cancer. 2003;103:647-51.

7. Hofmann H-S, Hansen G, Riehter G, Taege C, Simm A, Silber R-E, et al. Matrix metalloproteinase-12 expression correlates with local recurrence and metastatic disease in non-small cell lung cancer patients. Clin Cancer Res. 2005;11:1086-92.

8. Heist RC, Marshall AL, Liu G, Zhou W, Su L, Neuberg D, et al. Matrix metalloproteinase polymorphisms and survival in stage I non-small cell lung cancer. Clin Cancer Res. 2006; 12:5448-53.

9. Passlick B, Sienel W, Seen-Hibler R, Wöckel W, Thetter O, Mutschler W, et al. Overexpression of matrix metalloproteinase 2 predicts unfavorable outcome in early-stage non-small cell lung cancer. Clin Cancer Res. 2000;6:3944-8.

10. Pinto CA, de Oliveira Carvalho PE, Antonângelo L, Garippo A, da Silva AG, Soares F, et al. Morphometric evaluation of tumor matrix metalloproteinase 9 predicts survival after surgical resection of adenocarcinoma of the lung. Clin Can cer Res. 2003;9:3098-104.

11. Thomas P, Khokha R, Shepherd FA, Feld R, Tsao M-S. Differential expression of matrix metalloproteinases and their inhibitors in non-small cell lung cancer. J Pathol. 2000;190:150-6.

12. Liu HL, Zhang T, Li X, Huang J, Wu B, Huang X, et al. Predictive value of MMP7 expression for response to chemotherapy and survival in patients with nonsmall cell lung cancer. Cancer Sci. 2008;99:2185-92.

13. Overall CM, Kleifeld O. Validating matrix metalloproteinases as drug targets and anti-targets for cancer therapy. Nat Rev Cancer. 2006;6:227-39.

14. Leighl NB, Paz-Ares L, Douillard J-Y, Peschel C, Arnold A, Depierre A, et al Randomized phase III study of matrix metalloproteinase inhibitor BMS-275291 in combination with paclitaxel and carboplatin in advanced non-small lung cancer: National Cancer Institute of Canada-Clinical Trials Group Study BR.18. J Clin Oncol. 2005;23:2831-9.

15. Bissett D, O'Byrne KJ, von Pawel J, Gatzemeier U, Price A, Nicolson M, et al. Phase III study of matrix metalloproteinase inhibitor prinomastat in non-small cell lung cancer. J Clin Oncol. 2005;23:842-9.

16. Coussens LM, Fingleton B, Matrisian LM. Matrix metalloproteinase inhibitors and cancer: trials and tribulations. Science. 2002;295:2387-92.

17. Ikonomidis JS, Jones JA, Barbour JR, Stroud RE, Clark LL, Kaplan BS, et al Expression of matrix metalloproteinases and endogenous inhibitors within ascending aortic aneurysms of patients with Marfan Syndrome. Circulation. 2006;114(1 Suppl):I365-70.

18. Spinale FG, Coker ML, Heung LJ, Bond BR, Gunasinghe HR, Etoh T, et al. A matrix metalloproteinase induction/activation system exists in the human left ventricular myocardium and is upregulated in heart failure. Circulation. 2000;102: 1944-9.

19. Mukherjee R, Lowry AS, Allen RA, Stroud MR, Wharton JM, Ikonomidis JS, et al. Selective induction of matrix metalloproteinases and tissue inhibitor of metalloproteinases in atrial and ventricular myocardium in patients with atrial fibrillation. Am J Cardiol. 2006;97:532-7.

20. Deschamps AM, Yarbrough WM, Squires CE, Allen RA, Dowdy KB, McLean JE, et al. Trafficking of the membrane type-1 matrix metalloproteinase (MT1-MMP) in ischemia and reperfusion: relation to interstitial MT1-MMP activity. Circulation. 2005;111:1166-74.

21. Ford RL, Mains IM, Hilton EJ, Reeves ST, Stroud RE, Crawford FA, et al Endothelin-A receptor inhibition after cardiopulmonary bypass: cytokines and receptor activation. Ann Thorac Surg. 2008;86:1576-83.

22. Gottschalk PG, Dunn JR. The five-parameter logistic: a characterization and comparison with the four-parameter logistic. Anal Biochem. 2005;343:54-65.

23. Simi L, Andreani M, Davini F, Janni A, Pazzagli M, Serio M, et al. Simultaneous measurement of MMP9 and TIMP1 mRNA in human non small cell lung cancers by multiplex real time RT-PCR. Lung Cancer. 2004;45:171-9.

24. Safranek J, Pesta M, Holubec L, Kulda V, Dreslerova J, Vrzalova J, et al. Expression of MMP-7, MMP-9, TIMP-1 and TIMP-2 mRNA in lung tissue of patients with non-small cell lung cancer (NSCLC) and benign pulmonary disease. Anticancer Res. 2009;29:2513-7.

25. Buergy D, Weber T, Maurer GD, Mudduluru G, Medved F, Leupold JH, et al Urokinase receptor, MMP-1 and MMP-9 are markers to differentiate prognosis, adenoma and carcinoma in thyroid malignancies. Int J Cancer. 2009;125: 894-901.

26. Gentner B, Wein A, Croner RS, Zeittraeger I, Wirtz RM, Roedel F, et al. Differences in the gene expression profile of matrix metalloproteinases (MMPs) and their inhibitors (TIMPs) in primary colorectal tumors and their synchronous liver metastases. Anticancer Res. 2009;29:67-74.

27. Overall CM, Kleifeld $\mathrm{O}$. Towards third generation matrix metalloproteinase inhibitors for cancer therapy. Br J Cancer. 2006;94:941-6.

28. Munshi HG, Stack MS. Reciprocal interactions between adhesion receptor signaling and MMP regulation. Cancer Metastasis Rev. 2006;25:45-56.

29. Clark IM, Swingler TE, Sampieri CL, Edwards DR. The regulation of matrix metalloproteinases and their inhibitors. Int J Biochem Cell Biol. 2008;40:1362-78.

30. Tutton MG, George ML, Eccles SA, Burton S, Swift RI, Abulafi AM. Use of plasma MMP-2 and MMP-9 levels as a surrogate for tumour expression in colorectal cancer patients. Int J Cancer. 2003;107:541-50.

31. Langenkiöld M, Holmdahl L, Falk P, Ivarsson M-L. Increased plasma MMP-2 protein expression in lymph node-positive patients with colorectal cancer. Colorectal Dis. 2005;20:245-52.

32. Ranuncolo SM, Armanasco E, Cresta C, Bal De Kier, Joffe E, Puricelli L. Plasma MMP-9 (92 kDa-MMP) activity is useful in the followup and in the assessment of prognosis in breast cancer patients. Int J Cancer. 2003;106:745-51. 
33. Wu C-Y, Wu M-S, Chiang E-P, Chen Y-J, Chen C-J, Chi N-H, et al. Plasma matrix metalloproteinase-9 level is better than serum matrix metalloproteinase- 9 level to predict gastric cancer evolution. Clin Cancer Res. 2007;13:2054-60.

34. Ramankulov A, Lein M, Johannsen M, Schrader M, Miller K, Jung K. Plasma matrix metalloproteinase-7 as a metastatic marker and survival predictor in patients with renal cell carcinoma. Cancer Sci. 2008;99:1188-94.

35. Iizasa T, Fujisawa T, Suzuki M, Motohashi S-I, Yasufuku K, Yasakawa T, et al. Elevated levels of circulating plasma matrix metalloproteinase-9 in non-small cell lung cancer patients. Clin Cancer Res. 1999;5:149-53.

36. Baker AH, Edwards DR, Murphy G. Metalloproteinase inhibitors: biological actions and therapeutic opportunities. J Cell Sci. 2002;115:3719-27.

37. Visse R, Nagase H. Matrix metalloproteinase and tissue inhibitors of metalloproteinases. Circ Res. 2003;92:827-39.

38. Aljada IS, Ramnath N, Donohue K, Harvey S, Brooks JJ, Wiseman SM, et al. Upregulation of the tissue inhibitor of metalloproteinase-1 protein is associated with progression of human non-small cell lung cancer. J Clin Oncol. 2004;22: 3218-29.

39. Gouyer V, Conti M, Devos P, Zerimech F, Copin M-C, Créme E, et al. Tissue inhibitor of metalloproteinase 1 is an independent predictor of prognosis in patients with nonsmall cell lung carcinoma who undergo resection with curative intent. Cancer. 2005;103:1676-84.

\section{Discussion}

Dr David R. Jones (Charlottesville, $\mathrm{Va}$ ). I have only a few questions for you. You looked only at protein levels of the MMPs. Can you say what you perceive are some of the pitfalls of looking at these in isolation in a small sample size, and do you believe that perhaps looking at messenger RNA levels would add some value to your analysis?
Dr Shah. Thank you for your question. Yes, we acknowledge that we do have a small sample size, but our assay is very sensitive and highly quantifiable even in small samples, as we have previously shown in cardiovascular studies. We decided to look at the protein because it is the final effector and, therefore, our focus.

Dr Jones. Second, several groups have shown that MMP-9 levels are predictive of recurrence, much as you have seen here. I found it interesting that there was no difference in MMP-9 levels between your normal and your cancerous lung tissue. Do you have a possible explanation for that?

Dr Shah. The difference may be that semiquantitative methods were used in previous studies. It is also known that MMP may be a marker of greater importance in more advanced NSCLC.

Dr Dao M. Nguyen (Miami, Fla). You used a tumor homogenate. It is a mixture of cells from the tumor itself and from the stroma. Are the MMPs coming from the stroma, like tumor-derived stromal tissues, or are they coming from the cancer cells themselves, or both?

Dr Shah. That is a good question. I honestly could not speculate. I would assume they are coming from the tumor just because of the difference between the tumor sample and the normal sample.

Dr Nguyen. It can come from both, actually. You should look into it. I think laser-capture microdissection can help you with that.

Second question: This is a protein and these are enzymes. Are you planning to do enzyme assays? A lot of these proteins are present in small quantity and hard to detect. Do you plan to do zymograms?

Dr Shah. Possibly in future studies, yes. 